\title{
Fulminant Myocarditis Complicated with Immune Checkpoint Inhibitor in Patients with Thymoma: Report of Three Cases and Literature Review
}

\section{Daozheng Huang}

Guangdong Provincial People's Hospital

Feier Song ( $\square$ feiersong@126.com )

Guangdong Provincial People's Hospital https://orcid.org/0000-0003-0881-1249

\section{Weidai Zhang}

Shantou Central Hospital

\section{Huan Ma}

Guangdong Provincial People's Hospital

\section{Xingtao Lin}

Guangdong Provincial People's Hospital

\section{Tiehe Qin}

Guangdong Provincial People's Hospital

\section{Shouhong Wang}

Guangdong Provincial People's Hospital

\section{Case report}

Keywords: myocarditis, immune checkpoint inhibitors, programmed cell death protein 1 inhibitor, PD-1 inhibitor, thymoma

Posted Date: August 4th, 2021

DOI: https://doi.org/10.21203/rs.3.rs-707559/v1

License: (a) (1) This work is licensed under a Creative Commons Attribution 4.0 International License. Read Full License 


\section{Abstract}

Introduction

Immune checkpoint inhibitors (ICls) prevent the immune escape of tumor cells, which is beneficial to immune cells such as T lymphocytes to continuously monitor and kill tumor cells, and ultimately play an effective role in anti-tumor. ICl-associated myocarditis (irMyositis) was rarely reported, but its onset appeared latent, rapidly developed, and lethal, which has gradually attracted the attention of clinicians.

Case Report

We reported cases of fulminant myocarditis in 3 patients with thymoma who were treated with pembrolizumab, one of the programmed cell death protein 1 inhibitors (PD-1 inhibitors).

Management \& Outcome

The 3 patients were treated with methylprednisolone, immunoglobulin. Temporary pacemaker implantation was performed when case 1 suffered atrioventricular dissociation and ventricular escape. Cardioversion was performed in case 2 for the onset of ventricular tachycardia. In case 3, Extra-Corporeal Membrane Oxygenation was implemented. Unfortunately, the heart function did not improve and was succeeded by multiple organ failure in all 3 cases. The family withdrew treatment and led to lethal outcomes.

Discussion

Although ICls have achieved encouraging results in anti-tumor, their adverse reactions cannot be ignored. Patients with thymoma are more prone to autoimmune reactions which are needed more attention 1 . We reported cases of immunotherapy-associated myocarditis in patients with previous PM, showing the clinical and pathological features in the specific populations.

\section{Introduction}

At present, there have been relatively few cases in which thymoma be treated with ICls and its corresponding adverse reactions remain known. Patients with thymoma are more prone to autoimmune reactions which are needed more attention ${ }^{1}$. We reported 3 cases of unexpected, lethal myocarditis after immunotherapy in patients with thymoma, which helped to increase evidence and clinical experience. Moreover, the pathological features of the myocardium and skeletal muscle in patients with thymoma and complicated with immunotherapy-associated myocarditis were rarely reported. To our knowledge, this was the first time to report pathological results and could help to compare different types of cancers. Most of the current clinical trials have excluded cases with previous autoimmune diseases, leading to the unclear safety endpoints of immunotherapy in patients with a history of autoimmune diseases. We reported cases of immunotherapy-associated myocarditis in patients with previous PM, showing the clinical and pathological features in the specific populations. 


\section{Case Reports}

\section{Case 1}

The patient was a 43-year-old male, who had been physically healthy. On August 12,2015 , he underwent mediastinal tumor resection in Guangdong Provincial People's Hospital. The postoperative pathological diagnosis was "thymoma, type B2", and the patient received the chemotherapy with cyclophosphamide plus cisplatin. On September 18, 2016, the contrast-enhanced chest CT scan revealed a recurrence of thymoma with left pleural metastasis. On September 23, 2016, a mediastinal mass resection was performed. After the surgery, the patient received chemotherapy with Docetaxel plus Lobaplatin. In July 2018, limb weakness was found in the patient and he was admitted into the hospital. On admission, serum levels of creatine kinase (CK) (6370 U/L), Creatine Kinase-MB Isoenzyme (CK-MB) (97.7 U/L), and troponin T (TNT) $(571.8 \mathrm{pg} / \mathrm{ml})$ increased significantly. Left ventricular ejection fraction (LVEF) was $73 \%$, estimated by echocardiography on July 13,2018 . Moreover, biomarkers of vasculitis such as ANCAnuclear, antinuclear Antibody ANA was positive. Electromyography (EMG) suggested partial myogenic damage. the patient was diagnosed with polymyositis (PM), and the symptom alleviated after treatment of glucocorticoid. Meanwhile, the chest CT indicated the thymoma metastasis to the left thoracic cavity and therefore surgery was performed. Later, multiple CT scans performed in 2019 showed that thymoma was progressing. Accordingly, after the routine examination such as the electrocardiogram (ECG) showing substantially normal, on June 24,2019 , the patients received chemotherapy with pembrolizumab (200mg) plus paclitaxel (300mg).

On July 2, 2019, symptoms of chest tightness, shortness of breath, and limb weakness showed up. The myocardial enzyme tested in the community hospital was elevated. Chest CT scan showed that the pleural metastasis lesion was reduced. The symptoms did not alleviate after the patient was given glucocorticoid (methylprednisolone) and diuretics. Moreover, on July 6, 2019, tracheal intubation and ventilator-assisted ventilation were performed due to dyspnea and respiratory failure. The patient was transferred to Guangdong Provincial Peoples Hospital for intensive care and further treatment on July 7 , 2019.

Physical examination on admission: Temperature $(T): 36.5^{\circ} \mathrm{C}$, Pulse $(P): 84$ beats per min, Respiratory rate (R): 20 times per min, Blood pressure (BP): 122 / $80 \mathrm{mmHg}$. The patient was conscious. There was no rash on the skin or mucosa. The lungs were clear with no pleural rub. The heart rate (HR) was 80 beats per min with sinus rhythm. No pathological murmur or pericardial friction rubs were detected through auscultation. The limbs were not swollen.

Auxiliary examination:

A. CK: 5806 U/L; CK-MB: 193.5 U/L; peak high sensitive troponin T (HS-TNT): > 10000 pg/ml; peak N terminal pro B type natriuretic peptide (NT-proBNP): 7140 (pg/ml); positive acetylcholine receptor antibody ( $>20 \mathrm{nmol} / \mathrm{L}$, normal range $<0.45 \mathrm{nmol} / \mathrm{L}$ ); positive ryanodine receptor calcium release 
channel receptor antibody (normal range: negative); negative idiopathic inflammatory myopathy panel; negative paraneoplastic syndrome panel.

B. ECG: atrioventricular dissociation, multi-source ventricular escape rhythm, and HR were $60-80$ beats per min.

C. Echocardiography: LVEF was $55 \%$.

D. Chest X-ray: infections of lungs on both sides.

E. Muscle biopsy of the right quadriceps: fragmentary specimens, fibrous tissue, fat, and muscle tissue.

F. Myocardial biopsy: Fig. 1.

Diagnosis: 1, acute severe myocarditis, arrhythmia, heart failure, New York Heart Association cardiac function grade IV; 2 , suspected acute coronary syndrome; 3, PM; 4, suspected myasthenia gravis; 5 , thymic malignant tumor (recurrence)

Intervention: methylprednisolone $80 \mathrm{mg} /$ day; immunoglobulin 25g/day; Pyridostigmine Bromide 60mg q6h. Shortness of breath was slightly alleviated after treatment. However, on July 9, 2019, the patient suffered atrioventricular dissociation, ventricular escape beat with the HR of 35-50 beats per min. Temporary pacemaker implantation was performed and coronary angiography showed no occlusion or slow flow (TIMI level 2) of the vessels. Within 24 hours, cardiac arrest, ventricular tachycardia, and ventricular fibrillation showed up repeatedly and all rescue measures proved ineffectual.

Prognosis: death.

Naranjo score 2 : 2 (Table S1)

\section{Case 2}

The patient was a 27-year-old female, who had been physically healthy and denied a history of smoking. On May 12, 2019, a contrast-enhanced chest CT scan revealed a thymic tumor in the anterior superior mediastinum with pleural metastasis. The pleural biopsy indicated thymoma (type B2, stage IV with pleural metastasis. On June 5, 2019, the patient received chemotherapy with albumin-bound Paclitaxel (200mg), carboplatin (740mg) and pembrolizumab (200mg). On June 17, 2019, the patient started to suffer from limb weakness, shortness of breath, right eyelid ptosis, and stress urinary incontinence. The symptoms gradually worsened, and the patient was transferred to the intensive care unit on June 21, 2019.

Physical examination: T: $36.2^{\circ} \mathrm{C}, \mathrm{P}: 150$ beats per min, R: 20 times per min, BP: 124 / $89 \mathrm{mmHg}$. The patient was conscious. There was no rash on the skin or mucosa. The lungs were clear with no rales or pleural rub. The HR was 134 beats per min with sinus rhythm. No pathological murmur or pericardial friction rubs were detected through auscultation. The limbs were not swollen. 
Auxiliary examination:

A. on admission: CK: 10988 U/L; CK-MB: 293.7 U/L; peak HS-TNT: 6558.0 pg/ml; peak NT-proBNP: 4030.0 $\mathrm{pg} / \mathrm{ml}$.

B. the ECG showed second-degree atrioventricular blocks: Mobitz type I, intraventricular aberrant conduction. The arrhythmia aggravated ventricular tachycardia later.

C. Echocardiography: LVEF was $41 \%$.

Diagnosis: 1, acute severe myocarditis, arrhythmia, heart failure, New York Heart Association cardiac function grade IV; 2 , suspected myasthenia gravis; 3 , suspected myositis; 4, thymoma (type B2, stage IV with pleural metastasis).

Intervention: the patient was treated with methylprednisolone $80 \mathrm{mg} /$ day intravenously. The onset of ventricular tachycardia started on the day of admission with the ventricular HR of 200-250 beats per min, accompanied by decreasing blood pressure. Cardioversion helped in converting to sinus rhythm. Continuous renal replacement therapy (CRRT) was initiated on the same day due to anuria. However, the patient suffered from frequent premature ventricular contraction and recurrent ventricular tachycardia. Lidocaine was used as antiarrhythmics and electrolyte disturbance was remedied. The dose of methylprednisolone was increased to $120 \mathrm{mg}$ /day. Unfortunately, the patient developed a pulseless ventricular tachycardia on June 23,2019 , and cardiac arrest occurred after cardioversion. All rescue measures proved ineffectual and no return of spontaneous cardiac rhythm.

Prognosis: death.

Naranjo score: 2 (Table S1)

\section{Case 3}

The patient was a 52-year-old male, with a medical history of kidney stones and smoked for more than 30 years. In December 2017, mass in the lung was found through an annual health check. On March 27, 2018 , the CT scan found a right lung neoplasm with pleural metastasis. The lung biopsy showed thymoma (suspected type B1 or type AB). On April 19, 2018, the therapy of albumin-bound Paclitaxel (200 $\mathrm{mg}$ ), carboplatin (740 mg), and pembrolizumab (200 mg) were administered. On May 3, 2018, the patient developed fever, soreness, limb weakness, shortness of breath, and left eyelid ptosis. The patient was admitted into the community hospital on May 8, 2018. Tests on myocardial enzymes showed elevated CK (25612 U/L) and CK-MB (969 U/L), positive acetylcholine receptor antibody $(5.50 \mathrm{nmol} / \mathrm{L}$, normal range $<0.45 \mathrm{nmol} / \mathrm{L}$ ), positive ryanodine receptor calcium release channel receptor antibody (normal range: negative), and positive connexin antibody (normal range: negative). Chest CT scan showed a mass in the right lung, pneumonia on both sides. Between May 8, 2018, to May 10, 2018, the patient was treated with methylprednisolone $40 \mathrm{mg} /$ day intravenously, intravenous immune globulin (IVIG) $35 \mathrm{~g} /$ day, and antiinfection. On May 10, 2018, the patient developed a complete atrioventricular block with the HR 
decreasing to 24-28 beats per min. Emergent implantation of the temporary pacemaker was performed. Between May 11, 2018, to May 13, 2018, the dose of methylprednisolone was increased to $500 \mathrm{mg} /$ day, combined with IVIG 35g/day). The disease showed no improvement and on May 14, 2018, the patient was transferred to the intensive care unit in Guangdong Provincial People's Hospital.

Physical examination: T: $36.0^{\circ} \mathrm{C}, \mathrm{P}: 130$ beats per min, R: 25 times per min, BP: 78 / $58 \mathrm{mmHg}$. The patient was in a coma. There was no rash on the skin or mucosa. The pupils were equal and round with a diameter of $3 \mathrm{~mm}$ but no light reflex on both sides. The lungs were rough with moist rales of both lower lungs. No pleural rub was heard. The HR was 130 beats per min with sinus rhythm. No pathological murmur or pericardial friction rubs were detected through auscultation. The limbs were not swollen.

Auxiliary examination:

A. on admission: CK: 7633 U/L, CK-MB: 159.5 U/L. peak HS-TNT: > 10000 pg/ml; peak NT-proBNP: 5696 $\mathrm{pg} / \mathrm{ml}$.

B. ECG: complete atrioventricular block.

C. Echocardiography: LVEF was $21 \%$.

Diagnosis: 1. acute severe myocarditis arrhythmia Heart failure, New York Heart Association cardiac function grade IV; 2 , myasthenia gravis; 3 . Suspected myositis; 4 . Thymoma (type B1 or type AB)

Intervention: the patient was treated with methylprednisolone $40 \mathrm{mg} /$ day intravenously, IVIG $35 \mathrm{~g} /$ day, and anti-infection. Within 24 hours since admission, ventricular escape beat emerged with an HR of approximately 30 beats per min, accompanied by the loss of blood pressure and oxygen saturation. After cardiopulmonary resuscitation, the patients returned to sinus rhythm but no spontaneous circulation. Extra-Corporeal Membrane Oxygenation was implemented. However, the heart function did not improve and was succeeded by multiple organ failure. The family withdrew from treatment on May 15, 2018.

Prognosis: multiple organ dysfunction syndrome and withdrawal of treatment.

Naranjo score: 2 (Table S1)

\section{Discussion}

Immunotherapy is one of the effective methods for tumor treatment. The successful clinical application of ICls has led tumor treatment into a new era. However, the side effects remained unnoticed. The incidence of myocarditis after immunotherapy reported by Ganatra and Neilan was $0.06-1 \% 3,4$. Immunotherapy-associated fulminant myocarditis was rare but progressed rapidly, resulting in high mortality and poor prognosis ${ }^{5-7}$. ICls can be used in a variety of tumors, such as melanoma and nonsmall cell lung cancer ${ }^{7}$. The case reports of immunotherapy-associated myocarditis were mostly based on these two types of tumors ${ }^{6,8-11}$. 
Several recent Phase II clinical trials showed a therapeutic effect of pembrolizumab in patients with thymoma ${ }^{12,13}$. The chemotherapy regimen of pembrolizumab combined with albumin-bound Paclitaxel was promoted worldwide ${ }^{12}$. However, the thymus participated in the maturation of $\mathrm{T}$ lymphocytes, and patients with thymoma were more likely to have autoimmune diseases such as myasthenia gravis, nephritic nephrotic syndrome, rheumatoid arthritis, and lupus erythematosus ${ }^{12}$. Studies indicated that the incidence of immune-related adverse events (irAEs) in patients with thymoma after immunotherapy was significantly higher ${ }^{12,13}$. Three cases of immunotherapy-associated myocarditis were reported previously with a favorable prognosis. However, there were no detailed case reports of myocarditis after immunotherapy in patients with thymoma. Here, we reported 3 cases of fulminant myocarditis after chemotherapy of pembrolizumab plus paclitaxel, to discuss the risks of immunotherapy in patients with thymoma.

The diagnosis of myocarditis was based on the symptoms, non-ischemic cardiac structural dysfunction or myocardial damage, cardiac magnetic resonance, and endocardial biopsy ${ }^{14}$. Case 1 showed typical symptoms with significantly elevated troponin. Myocardial biopsy suggested inflammatory cell infiltration, myocardial cell degeneration, and non-ischemic necrosis. The onset of case 2 and case 3 was heart failure and arrhythmia, accompanied by elevated troponin. Echocardiography suggested systolic dysfunction. Moreover, the onset time of 3 cases was 8 to 15 days after immunotherapy, with no evidence of previous respiratory or digestive virus infection, which was consistent with the average onset time of 7 to 34 days reported by other studies $6,8,15,16$.

The risk factors for acute myocarditis after immunotherapy remains unclear ${ }^{13,15}$. Studies have found that baseline systolic function and electrocardiography did not predict immunotherapy-associated myocarditis ${ }^{8}$. A multicenter, case-control study, enrolling patients with melanoma and lung cancer, found that diabetes, sleep apnea-hypopnea syndrome, and obesity might be risk factors for myocarditis after immunotherapy ${ }^{8,17}$. However, the three cases reported in our study did not have any abovementioned factors, nor did they suffer from hypertension, hyperlipidemia, or previous heart disease. These patients did not prescribe the statin, radiotherapy, and with no evidence of cardiac metastasis. It might be suggested that the risk factors for fulminant myocarditis after thymoma were different from other tumors. In particular, Case 1 had a history of PM before receiving immunotherapy, suggesting that it was already autoimmune dysfunction before the patient received immunotherapy. Studies have found that patients with thymoma were more likely to develop autoimmune diseases after receiving immunotherapy, with PM and myocarditis being the most common complications ${ }^{13}$. The pathological feature of PM is that the CD8 + T lymphocytes invade non-necrotic muscle fibers, where T lymphocyte-mediated immune responses play an important role ${ }^{18}$. Myocardial histopathology of myocarditis caused by immunotherapy is also characterized by $T$ lymphocyte infiltration, suggesting that there may be an intersection of autoimmune damage. A retrospective study, published in 2018, found that myositis and myocarditis were co-morbidities when referring to irAEs, implying a similar or shared pathogenesis ${ }^{7}$. Furthermore, in the three cases of fulminant myocarditis in our study, PM was diagnosed since CK was significantly elevated. Accordingly, we believed that for patients with previous autoimmune diseases, especially those with a 
history of PM, immunotherapy might be risky for the higher probability to develop immune-associated myocarditis. Most of the current clinical trials have excluded cases with previous autoimmune diseases, leading to the unclear safety endpoints of immunotherapy in patients with a history of autoimmune diseases ${ }^{9,13,17,19}$.

In our report, the baseline characteristics of immune-associated myocarditis in 3 patients with thymoma were similar to those of other tumors ${ }^{6,17}$. Immunotherapy-associated myocarditis developed shortly after immunotherapy. The incidence of congestive heart failure, cardiogenic shock, and malignant arrhythmia after fulminant myocarditis was substantial ${ }^{20,21}$. The onset symptoms in our report were consistent with previous results. Studies reported that heart block in fulminant myocarditis was a predictor of higher mortality 20,22 . Likewise, cases in our study were complicated with heart block, suggesting that the predictive value of heart block applied in patients with thymoma. Interestingly, although case 1 had the symptoms of heart failure, the Echocardiography indicated a preserved LVEF, even during the deterioration, which was consistent with previous case reports $6,17,22$. Therefore, we believed that immunotherapy-associated myocarditis had different characteristics from viral myocarditis. It might not appropriate to estimate with only LVEF. A comprehensive examination of myocardial enzymes, autoantibodies, and multiple organ functions helped with the optimal treatment ${ }^{23}$.

In particular, patients with thymoma were prone to immune disorders, especially myasthenia gravis ${ }^{24}$. In our study, all the 3 cases reported new-onset limb weakness with ptosis and were finally diagnosed with myasthenia gravis. However, we could not confirm the etiology though we suspected it was caused by thymoma or PD-1 inhibitor (pembrolizumab) or both. Moreover, the case-control study was needed to estimate if myasthenia gravis could be a marker of poor prognosis.

PD-1 gene was mainly expressed on the surface of activated T cells. When it bound to PD-L1 on the surface of tumor cells, it down-regulated the activity of cytotoxic T cells, so that tumor cells avoided immune surveillance ${ }^{12,25}$. ICls (such as pembrolizumab) enhanced the immune surveillance and immune clearance of $T$ cells by inhibiting the binding of PD- 1 to PD-L $1^{26}$. The mechanism of ICl-associated acute myocarditis was still unclear ${ }^{20}$. In mice, PD-1 functioned in the myocardial immune response and inhibits inflammatorily cardiomyocyte injury ${ }^{27}$. On the contrary, dilated cardiomyopathy in PD-1 gene-deficient mice explained the effect of PD-1 on protecting cardiomyocytes due to the production of autoimmune antibodies against troponin I (TNI) $)^{28,29}$.

Previous studies found T lymphocytes- and/or macrophages-infiltrated myocardial tissue in patients with immunotherapy-associated myocarditis $5,6,8,22$, which was consistent with the result of myocardial biopsy in our study. It indicated similar pathogenic and pathological characteristics in thymoma, compared with other tumors.

At present, glucocorticoid pulse therapy was the main treatment of $\mathrm{ICl}$-associated myocarditis ${ }^{7,30}$. Mahmood et al. summarized 35 cases of immunotherapy-associated myocarditis and found that high- 
dose glucocorticoid pulse therapy reduced the incidence of major adverse cardiac events (MACE) ${ }^{8}$. Besides, the IVIG can block autoimmune antibodies and inhibit autoimmune reactions, which has been widely used in treating viral myocarditis and rheumatic immune diseases. However, Cases 1 and 3 in our study did not recover after glucocorticoid plus IVIG therapy. Mahmood et al. also reported 2 cases treated with IVIG with one death and one survival ${ }^{8}$. Also, Salem et al. reported a successful case of fulminant myocarditis after nivolumab (a PD-1 inhibitor) with Abatacept (a CTLA-4 agonist) in a patient with terminal lung cancer ${ }^{4}$. Esfahani et al. reported a case of melanoma complicating fulminant myocarditis after immunotherapy and treated with alemtuzumab ${ }^{31}$. Abatacept is mainly recommended for rheumatoid arthritis and Alemtuzumab for chronic lymphocytic leukemia and multiple sclerosis. There was no evidence of their safety and effectiveness.

Frigeri et al. reported a case of early use of ECMO and IABP on fulminant myocarditis after immunotherapy ${ }^{21}$. In our study, case 3 received ECMO and IABP only after repeated cardiac arrest and cardiopulmonary resuscitation. Whether early use of heart-assist devices such as IABP, Impella, or ECMO helped in the treatment required further study.

\section{Conclusion}

ICls provide a new approach for patients with advanced cancer, including thymoma. However, irAEs after immunotherapy, especially fulminant myocarditis, cause high mortality. We reported 3 cases of thymoma and found that the symptoms of irAEs were atypical, with acute progression, poor prognosis, and limited treatments. Further studies are needed to better understand the pathogenesis, identify high-risk patients with biomarkers, and explore the optimal treatment.

\section{Declarations}

\section{Ethics approval and consent to participate}

The study was conducted in accordance with the Declaration of Helsinki and was approved by the institutional ethics committee. Written informed consent was obtained from each patient. In all cases, the authors have obscured the identity of the deceased.

\section{Consent for publication}

Not applicable.

\section{Availability of data and materials}

All data generated or analyzed during this study are included in this published article.

\section{Competing Interests}

The authors declare that they have no conflict of interest. 


\section{Funding}

This work was supported by the grant from Medical Scientific Research Foundation of Guangdong Province, Guangdong, People's Republic of China. (Grant number: A2018064)

\section{Authors' Contributions}

Daozheng Huang, Weidai Zhang and Xingtao Lin contributed to patients care. Feier Song drafted the manuscript. Huan Ma revised the manuscript. Shouhong Wang and Tiehe Qin conceived of the study and participated in its design and coordination and helped to draft the manuscript. All authors read and approved the final manuscript.

\section{Acknowledgements}

The authors confirm that the Principal Investigator for this paper is Shouhong Wang and that she had direct clinical responsibility for patients.

\section{References}

1. Touat M, Maisonobe T, Knauss $S$, et al. Immune checkpoint inhibitor-related myositis and myocarditis in patients with cancer. Neurology. 2018;91:e985-94.

2. Naranjo CA, Busto U, Sellers EM, et al. A method for estimating the probability of adverse drug reactions. Clin Pharmacol Ther. 1981;30:239-45.

3. Ganatra S, Neilan TG. Immune Checkpoint Inhibitor-Associated Myocarditis. Oncologist. 2018;23:879-86.

4. Salem JE, Allenbach Y, Vozy A, et al. Abatacept for Severe Immune Checkpoint Inhibitor-Associated Myocarditis. N Engl J Med. 2019;380:2377-9.

5. Norwood TG, Westbrook BC, Johnson DB, et al. Smoldering myocarditis following immune checkpoint blockade. J Immunother Cancer. 2017;5:91.

6. Johnson DB, Balko JM, Compton ML, et al. Fulminant Myocarditis with Combination Immune Checkpoint Blockade. N Engl J Med. 2016;375:1749-55.

7. Wang DY, Salem JE, Cohen JV, et al. Fatal Toxic Effects Associated With Immune Checkpoint Inhibitors: A Systematic Review and Meta-analysis. JAMA Oncol. 2018;4:1721-8.

8. Mahmood SS, Fradley MG, Cohen JV, et al. Myocarditis in Patients Treated With Immune Checkpoint Inhibitors. J Am Coll Cardiol. 2018;71:1755-64.

9. Menzies AM, Johnson DB, Ramanujam S, et al. Anti-PD-1 therapy in patients with advanced melanoma and preexisting autoimmune disorders or major toxicity with ipilimumab. Ann Oncol. 2017;28:368-76.

10. Heinzerling L, Ott PA, Hodi FS, et al. Cardiotoxicity associated with CTLA4 and PD1 blocking immunotherapy. J Immunother Cancer. 2016;4:50. 
11. Escudier M, Cautela J, Malissen N, et al. Clinical Features, Management, and Outcomes of Immune Checkpoint Inhibitor-Related Cardiotoxicity. Circulation. 2017;136:2085-7.

12. Giaccone G, Kim C, Thompson J, et al. Pembrolizumab in patients with thymic carcinoma: a singlearm, single-centre, phase 2 study. Lancet Oncol. 2018;19:347-55.

13. Cho J, Kim HS, Ku BM, et al. Pembrolizumab for Patients With Refractory or Relapsed Thymic Epithelial Tumor: An Open-Label Phase II Trial. J Clin Oncol. 2019;37:2162-70.

14. Caforio AL, Pankuweit S, Arbustini E, et al: Current state of knowledge on aetiology, diagnosis, management, and therapy of myocarditis: a position statement of the European Society of Cardiology Working Group on Myocardial and Pericardial Diseases. Eur Heart J 34:2636-48, 2648a2648d, 2013.

15. Moslehi JJ, Salem JE, Sosman JA, et al. Increased reporting of fatal immune checkpoint inhibitorassociated myocarditis. Lancet. 2018;391:933.

16. Anquetil C, Salem JE, Lebrun-Vignes B, et al. Immune Checkpoint Inhibitor-Associated Myositis. Circulation. 2018;138:743-5.

17. Tocchetti CG, Galdiero MR, Varricchi G. Cardiac Toxicity in Patients Treated With Immune Checkpoint Inhibitors: It Is Now Time for Cardio-Immuno-Oncology. J Am Coll Cardiol. 2018;71:1765-7.

18. Dalakas MC, Hohlfeld R. Polymyositis and dermatomyositis. Lancet. 2003;362:971-82.

19. Varricchi G, Galdiero MR, Tocchetti CG. Cardiac Toxicity of Immune Checkpoint Inhibitors: CardioOncology Meets Immunology. Circulation. 2017;136:1989-92.

20. Atallah-Yunes SA, Kadado AJ, Kaufman GP, et al. Immune checkpoint inhibitor therapy and myocarditis: a systematic review of reported cases. J Cancer Res Clin Oncol. 2019;145:1527-57.

21. Frigeri M, Meyer P, Banfi C, et al: Immune Checkpoint Inhibitor-Associated Myocarditis: A New Challenge for Cardiologists. Can J Cardiol 34:92 e1-92 e3, 2018.

22. Pradhan R, Nautiyal A, Singh S. Diagnosis of immune checkpoint inhibitor-associated myocarditis: A systematic review. Int J Cardiol. 2019;296:113-21.

23. Ammirati E, Cipriani M, Lilliu M, et al. Survival and Left Ventricular Function Changes in Fulminant Versus Nonfulminant Acute Myocarditis. Circulation. 2017;136:529-45.

24. Bernard $\mathrm{C}$, Frih $\mathrm{H}$, Pasquet $F$, et al. Thymoma associated with autoimmune diseases: 85 cases and literature review. Autoimmun Rev. 2016;15:82-92.

25. Keir ME, Butte MJ, Freeman GJ, et al. PD-1 and its ligands in tolerance and immunity. Annu Rev Immunol. 2008;26:677-704.

26. Pardoll DM. The blockade of immune checkpoints in cancer immunotherapy. Nat Rev Cancer. 2012;12:252-64.

27. Tarrio ML, Grabie N, Bu DX, et al. PD-1 protects against inflammation and myocyte damage in T cellmediated myocarditis. J Immunol. 2012;188:4876-84.

28. Nishimura H, Okazaki T, Tanaka Y, et al. Autoimmune dilated cardiomyopathy in PD-1 receptordeficient mice. Science. 2001;291:319-22. 
29. Okazaki T, Tanaka Y, Nishio R, et al. Autoantibodies against cardiac troponin I are responsible for dilated cardiomyopathy in PD-1-deficient mice. Nat Med. 2003;9:1477-83.

30. Haanen J, Carbonnel F, Robert C, et al. Management of toxicities from immunotherapy: ESMO Clinical Practice Guidelines for diagnosis, treatment and follow-up. Ann Oncol. 2017;28:iv119-42.

31. Esfahani K, Buhlaiga N, Thebault P, et al. Alemtuzumab for Immune-Related Myocarditis Due to PD-1 Therapy. N Engl J Med. 2019;380:2375-6.

\section{Tables}


Table 1

Clinical characteristics of 3 patients with FM

\begin{tabular}{|c|c|c|c|}
\hline No. & Case 1 & Case 2 & Case 3 \\
\hline Age(years) & 43 & 27 & 52 \\
\hline Gender & Male & Female & Male \\
\hline Previous history & none & none & Kidney stone \\
\hline histological type & type B2 & type B2 & Type $B 1$ or type $A B$ \\
\hline therapeutic effect & $\begin{array}{l}\text { Pleural metastases } \\
\text { are slightly smaller }\end{array}$ & Not reviewed & Not reviewed \\
\hline $\begin{array}{l}\text { Duration between } \\
\text { hospital } \\
\text { admission and } \\
\text { onset (days) }\end{array}$ & 8 & 12 & 15 \\
\hline \multicolumn{4}{|l|}{ Symptoms } \\
\hline Limb weakness & + & + & + \\
\hline dyspnea & + & + & + \\
\hline ptosis & + & + & + \\
\hline \multicolumn{4}{|l|}{ Laboratory tests } \\
\hline Index CK(U/L) & 5806 & 10988 & 7633 \\
\hline Index CK-MB(U/L) & 193.5 & 293.7 & 159.5 \\
\hline Index CK-MB/CK & $3.33 \%$ & $1.76 \%$ & $2.09 \%$ \\
\hline Peak CK(U/L) & 7273 & 25862 & 7633 \\
\hline Peak CK-MB(U/L) & 192.5 & 359.6 & 433.4 \\
\hline $\begin{array}{l}\text { Peak } \\
\text { hsTNT(pg/ml) }\end{array}$ & $>10000$ & 6558.0 & $>10000$ \\
\hline $\begin{array}{l}\text { Peak NT- } \\
\text { proBNP(pg/ml) }\end{array}$ & 7140 & 4030.0 & 5696 \\
\hline MuSK.Ab & - & Not reviewed & - \\
\hline RyR.Ab & + & Not reviewed & + \\
\hline
\end{tabular}

CK, creatine kinase; CKMB, Creatine Kinase-MB; hsTNT, high-sensitive cardiac troponin T; NT-proBNP, $\mathrm{N}$ terminal pro B type natriuretic peptide; MuSK.Ab, anti-skeletal muscle receptor tyrosine kinase antibody; RyR.Ab, ryanodine receptor calcium release channel antibody; AchR.Ab, acetylcholine receptor antibody; Titin-Ab, connexin antibody / Human actin antibody; LVEF, left ventricular ejection fraction; irAEs, immune-related adverse events; CRRT, continuous renal replacement therapy; ECMO, Extra-Corporeal Membrane Oxygenation. 


\begin{tabular}{|c|c|c|c|}
\hline No. & Case 1 & Case 2 & Case 3 \\
\hline AchR.Ab & + & Not reviewed & + \\
\hline Titin-Ab & - & Not reviewed & - \\
\hline \multicolumn{4}{|l|}{$\begin{array}{l}\text { Auxiliary } \\
\text { examination }\end{array}$} \\
\hline Electrocardiogram & $\begin{array}{l}\text { atrioventricular } \\
\text { dissociation,multi- } \\
\text { source ventricular } \\
\text { escape rhythm }\end{array}$ & $\begin{array}{l}\text { second-degree atrioventricular } \\
\text { blocks: Mobitz type I, } \\
\text { intraventricular aberrant } \\
\text { conduction; refractory ventricular } \\
\text { tachycardia after disease } \\
\text { progression }\end{array}$ & $\begin{array}{l}\text { Complete } \\
\text { atrioventricular } \\
\text { block; }\end{array}$ \\
\hline $\begin{array}{l}\text { LVEF detected by } \\
\text { echocardiogram }\end{array}$ & $50 \%$ & $41 \%$ & $21 \%$ \\
\hline \multicolumn{4}{|l|}{ Biopsy } \\
\hline Myocardium & $\#$ & Not reviewed & Not reviewed \\
\hline Skeleton muscle & $\begin{array}{l}\text { Poor specimen } \\
\text { sampling }\end{array}$ & Not reviewed & Not reviewed \\
\hline \multicolumn{4}{|l|}{ irAEs } \\
\hline myocarditis & + & + & + \\
\hline polymyostitis & suspected & suspected & suspected \\
\hline $\begin{array}{l}\text { myasthenia } \\
\text { gravis }\end{array}$ & suspected & suspected & suspected \\
\hline \multicolumn{4}{|l|}{ Intervention } \\
\hline $\begin{array}{l}\text { Anti-inflammatory } \\
\text { agents }\end{array}$ & $\begin{array}{l}\text { methylprednisolone } \\
\text { and intravenous } \\
\text { immunoglobulin }\end{array}$ & methylprednisolone & $\begin{array}{l}\text { methylprednisolone } \\
\text { and intravenous } \\
\text { immunoglobulin }\end{array}$ \\
\hline $\begin{array}{l}\text { Advanced life } \\
\text { support }\end{array}$ & $\begin{array}{l}\text { mechanical } \\
\text { ventilation; } \\
\text { pacemaker }\end{array}$ & mechanical ventilation; CRRT & $\begin{array}{l}\text { mechanical } \\
\text { ventilation; } \\
\text { pacemaker; CRRT; } \\
\text { ECMO }\end{array}$ \\
\hline
\end{tabular}

CK, creatine kinase; CKMB, Creatine Kinase-MB; hsTNT, high-sensitive cardiac troponin T; NT-proBNP, $\mathrm{N}$ terminal pro B type natriuretic peptide; MuSK.Ab, anti-skeletal muscle receptor tyrosine kinase antibody; RyR.Ab, ryanodine receptor calcium release channel antibody; AchR.Ab, acetylcholine receptor antibody; Titin-Ab, connexin antibody / Human actin antibody; LVEF, left ventricular ejection fraction; irAEs, immune-related adverse events; CRRT, continuous renal replacement therapy; ECMO, Extra-Corporeal Membrane Oxygenation. 


\begin{tabular}{|c|c|c|c|}
\hline No. & Case 1 & Case 2 & Case 3 \\
\hline Outcome & $\begin{array}{l}\text { disease } \\
\text { progression and } \\
\text { withdrawal of } \\
\text { therapy }\end{array}$ & death & $\begin{array}{l}\text { multiple organ } \\
\text { dysfunction } \\
\text { syndrome and } \\
\text { withdrawal of } \\
\text { therapy }\end{array}$ \\
\hline \multicolumn{4}{|c|}{$\begin{array}{l}\text { CK, creatine kinase; CKMB, Creatine Kinase-MB; hsTNT, high-sensitive cardiac troponin T; NT-proBNP, } \\
\mathrm{N} \text { terminal pro B type natriuretic peptide; MuSK.Ab, anti-skeletal muscle receptor tyrosine kinase } \\
\text { antibody; RyR.Ab, ryanodine receptor calcium release channel antibody; AchR.Ab, acetylcholine } \\
\text { receptor antibody; Titin-Ab, connexin antibody / Human actin antibody; LVEF, left ventricular ejection } \\
\text { fraction; irAEs, immune-related adverse events; CRRT, continuous renal replacement therapy; ECMO, } \\
\text { Extra-Corporeal Membrane Oxygenation. }\end{array}$} \\
\hline
\end{tabular}

\section{Figures}
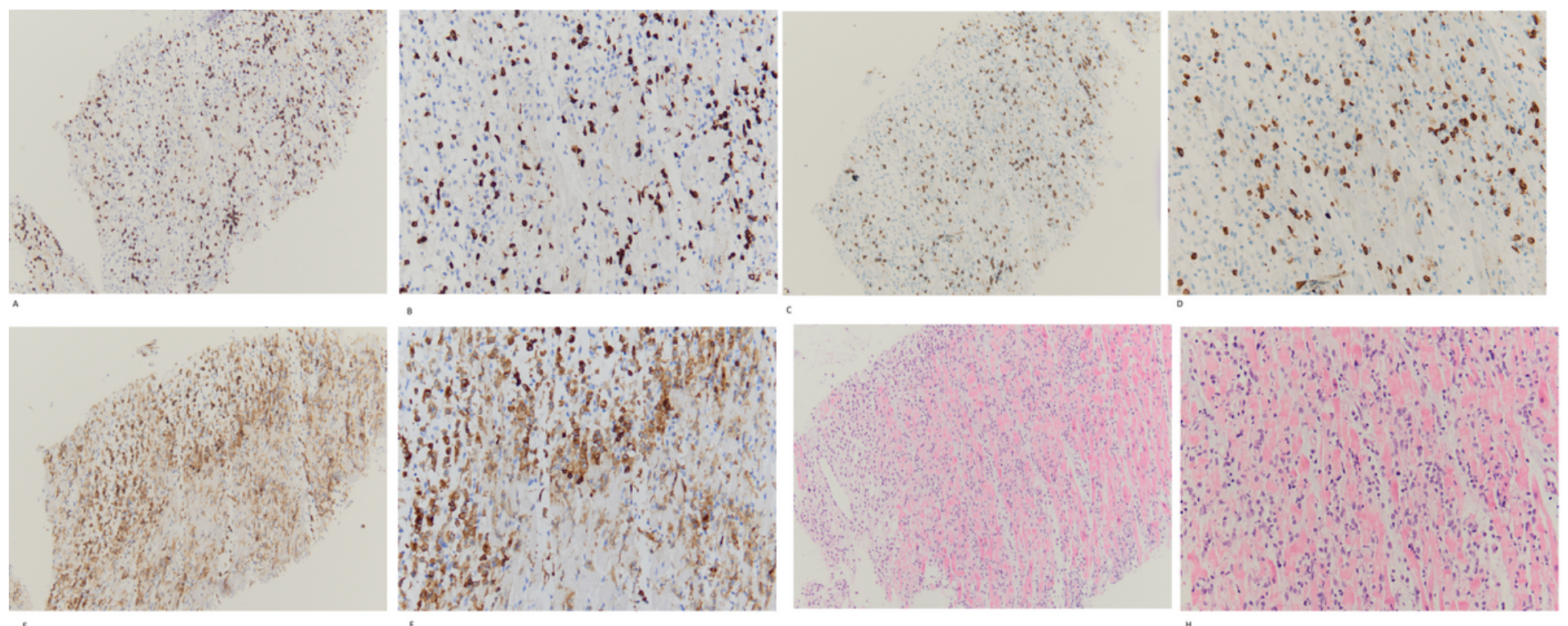

\section{Figure 1}

A. CD3-10X: T lymphocyte marker, distributed among cardiomyocytes, 10x B. CD3-20X: T lymphocyte marker, distributed among cardiomyocytes, 20x C. CD8-10X: Cytotoxic T lymphocyte subtype marker, distributed among cardiomyocytes, 10x D. CD8-20X: Cytotoxic T lymphocyte subtype marker, distributed among cardiomyocytes, 20x E. CD4-10X: helper T lymphocyte subtype marker, distributed among cardiomyocytes, 10x F. CD4-20X: helper T lymphocyte subtype marker, distributed among cardiomyocytes, 20x G. HE-10X: cardiomyocyte degeneration, necrosis, atrophy, interstitial lymphocyte infiltration, 10x H. HE-20X: cardiomyocyte degeneration, necrosis, atrophy, interstitial lymphocyte infiltration, $20 x$ 